\title{
Denosumab-Induced Medication-Related Osteonecrosis of the Jaw (DRONJ): A 5-Year Retrospective Cohort Study
}

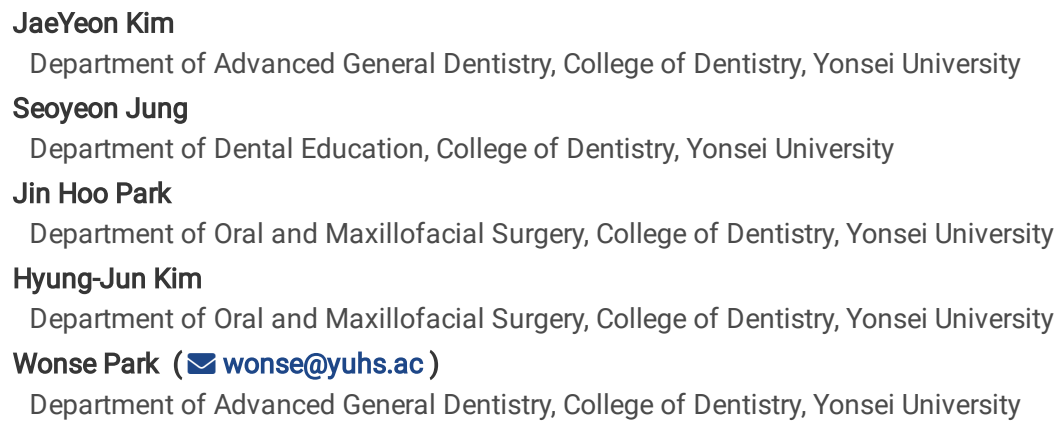

\section{Research Article}

Keywords: denosumab, osteoporosis, medication-related osteonecrosis of the jaw, denosumab-related osteonecrosis of the jaw

Posted Date: January 3rd, 2022

DOI: https://doi.org/10.21203/rs.3.rs-1186902/v1

License: @ (i) This work is licensed under a Creative Commons Attribution 4.0 International License. Read Full License 


\section{Abstract}

Denosumab (Dmab) has been suggested as a first-line therapy for osteoporotic patients. However, a standardized protocol for the prevention of Dmab induced medication-related osteonecrosis of the jaw (MRONJ) has not yet been established. Thus, we investigated the factors that can affect Dmab induced MRONJ (DRONJ) to elucidate the relationship between invasive dental treatment and Dmab administration in patients who underwent Dmab and invasive dental treatment (especially tooth extraction) between October 2016 and March 2020. Four of the 98 patients developed MRONJ before and after tooth extraction. Two out of 4 patients developed MRONJ regardless of invasive treatment after Dmab administration and proceeded with extraction, and one patient developed DRONJ after Dmab administration and extraction. The other patient underwent a tooth extraction without osteoporosis treatment, and spontaneous DRONJ developed after Dmab administration. All MRONJ/DRONJ cases reported in this study show that MRONJ/DRONJ can develop as chronic inflammation without invasive dental treatment, therefore, implementing preventive dental treatment before initiating Dmab treatment is necessary to reduce the likelihood of DRONJ.

\section{Introduction}

Bisphosphonates (BP) and the receptor activator of nuclear factor-KB (RANK) ligand (RANKL) inhibitor denosumab (Dmab) are the most common antiresorptive agents used in the treatment of osteoporosis ${ }^{1-3}$. Although Dmab has chiefly been used as a second-line therapy after BP, it was recently suggested as a first-line therapy for osteoporotic patients with a moderate or high risk of fracture 4 . However, Dmab must also be evaluated in light of the issues surrounding the use of bone resorption inhibitors, including BP. Particularly, medication-related osteonecrosis of the jaw (MRONJ) is one of the most controversial side effects of bone resorption inhibitors ${ }^{5,6}$.

MRONJ is defined based on three criteria: 1) history of ongoing or prior treatment with antiresorptive or antiangiogenic agents; 2) exposure of the jaw bone or exposed bone that can be probed through an intra-or extra-oral fistula in the maxillofacial region for >8 weeks; and 3) no history of radiotherapy or obvious metastases to the jaw ${ }^{7}$. Since BP-related osteonecrosis of the jaw (BRONJ) was first reported in 2003, it continues to be of interest to dental clinicians and researchers ${ }^{8,9}$. However, due to indipendent reports of Dmab-related bone necrosis, the American Association of Oral and Maxillofacial Surgeons (AAOMS) revised the term BRONJ to MRONJ in $2014^{7}$.

Various hypotheses have been put forth to explain why bone necrosis occurring after the administration of antiresorptive agents such as BP or Dmab is limited to the jaws. However, there is insufficient evidence regarding the exact pathogenesis of MRONJ ${ }^{10}$. The most important systemic risk factor for MRONJ is administration of a powerful anti-resorptive agent, such as a nitrogen-containing bisphosphonate or a RANKL inhibitor, and local risk factors include dentoalveolar surgery (especially tooth extraction), dentures, and existing inflammatory dental diseases (e.g., periodontal disease) ${ }^{7}$.

In 2009, based on sparse data, the AAOMS recommended a 3-month break from oral BPs before and after invasive dental procedures for osteoporotic patients who have used oral BPs for $>4$ years with one exception. That being that the removal of acutely-infected teeth in patients taking oral BPs should proceed immediately under robust antibiotic coverage. However, the AAOMS also found no evidence that interrupting BP therapy alters the risk of ONJ in patients after tooth extraction 7,11 . However, given the lack of standard duration of the drug break for MRONJ prevention in osteoporotic patients with a history of Dmab therapy, there is a need for retrospective clinical cohort studies to develop prevention guidelines and protocols for MRONJ in these patients.

Therefore, we investigated patients diagnosed with osteoporosis who were administered Dmab (Prolia $®$ ) at the Department of Endocrinology at Severance Hospital, and underwent invasive dental treatment (tooth extraction) at the Department of Advanced General Dentistry and Oral and Maxillofacial Surgery at Yonsei University Dental Hospital. Accordingly, the aim of this study was to investigate the factors that can affect DRONJ to elucidate the relationship between invasive dental treatment and Dmab administration and report several cases of DRONJ at our institution.

\section{Material And Methods Ethics statement}

This study protocol was approved by the Institutional Review Board (IRB) of the Yonsei University Dental Hospital (approval number: 2-2020-0071). Deidentified participant data were used and written informed consent was waived because the design of the study was retrospective study. This study was performed in accordance with the Declaration of Helsinki.

\section{Inclusion and Exclusion criteria}

We screened a total of 159 patients who received Dmab therapy at the Department of Endocrinology at Severance Hospital and underwent invasive dental treatment at the Department of Advanced General Dentistry and Oral and Maxillofacial Surgery at Yonsei University Dental Hospital from October 2016 to March 2020.

The inclusion criteria were as follows: age $>20$ years, diagnosis of osteoporosis, history of treatment with Dmab (60 mg administered as a single subcutaneous injection once every 6 months) or Dmab combined with BP (alendronate, ibandronate, pamidronate, risedronate, or zoledronate), and a history of invasive dental treatment (i.e., tooth extraction) at the Department of Advanced General Dentistry and Oral and Maxillofacial Surgery at Yonsei University Dental Hospital for teeth with hopeless prognosis due to various reasons, including caries, periodontitis, and impaction. The exclusion criteria were as follows: no history of Dmab therapy before and after invasive dental treatment, administration of Dmab 120 mg (Xgeva $\left.{ }^{\circledR}\right)$ instead of Dmab 60 mg (Prolia $\left.{ }^{\circledR}\right)$, history of other bone metabolism disorders or administered drugs, secondary osteoporosis due to oncological dose administration in cancer patients, history of Dmab 
therapy for diseases other than osteoporosis (e.g., hypercalcemia of malignancy, solid cancers, bone metastases, giant cell neoplasm, or multiple myeloma), and a history of Dmab or invasive dental therapy at a different institution, preventing accurate evaluation.

Overall, 98 (189 teeth) of the 159 patients initially screened were included in the study, after excluding 61 patients (108 teeth).

\section{Variables}

Participants' age, sex, oral condition, underlying disease, BP administration history, type of drug administered after tooth extraction, period from drug cessation to extraction, period from extraction to drug initiation, location of extraction (maxillary/mandibular/multiple) and presence or absence of MRONJ/DRONJ were investigated retrospectively.

For patients with MRONJ/DRONJ, the number of Dmab administrations, date of extraction, date of diagnosis, location, staging, associated local factors, and the type of MRONJ/DRONJ treatment (conservative/surgical) were additionally investigated. The prescription record was referred for information on Dmab administration, and electronic medical records were referred for information on the invasive dental treatment performed.

\section{Statistical analysis}

Patient demographics are expressed as $\mathrm{n}(\%)$ and mean \pm standard deviation. The $\chi^{2}$ test was used to compare proportions across levels of categorical variables. Due to the low incidence of MRONJ/DRONJ, 40 cases were randomly sampled to obtain stable results. To analyze possible associations between affecting factors with the development of MRONJ/DRONJ, we used a two-tailed Fisher's exact test. A two-tailed P-value $<0.05$ was considered statistically significant for all analyses. All statistical tests were performed using SPSS statistical software (SPSS for Windows, version 25; SPSS Inc., Chicago, IL, U.S.A).

\section{Results}

\section{Baseline characteristics}

The baseline characteristics of the 98 participants surveyed in this study, including the average age, sex, and the oral condition of the patients before tooth extraction are presented in Table 1. The participants' age ranged from 36 to 91 years, with an average age of $70.5 \pm 10.3$ years and the highest percentage of people in their 70s. There were 87 women (88.8\%). Residual roots, dental caries and other endodontic lesions were the main reasons for tooth extraction, followed by periodontitis, fracture/crack, and impacted/supernumerary tooth. Two patients (five teeth) underwent dental extraction due to osteonecrosis of the jaw.

Table 1

Demographics and clinical characteristics

\begin{tabular}{|c|c|}
\hline Characteristics & Study population \\
\hline Participants, n (\%) & $98(100)$ \\
\hline Age (years), mean (SD) & $70.5(10.31)$ \\
\hline \multicolumn{2}{|l|}{ Sex, n (\%) } \\
\hline Female & $87(88.8)$ \\
\hline Male & $11(11.2)$ \\
\hline \multicolumn{2}{|l|}{ Oral condition, patient $\mathrm{n}(\%)$ / teeth $\mathrm{n}(\%)$} \\
\hline Root rest / Dental caries / Endodontic lesion & $50(49.0) / 102(55.0)$ \\
\hline Periodontitis & $32(31.4) / 58(30.7)$ \\
\hline Fracture / Crack & $12(11.8) / 13(6.9)$ \\
\hline Impacted / Supernumerary tooth & $6(5.9) / 9(4.8)$ \\
\hline MRONJ & $2(2.0) / 5(2.6)$ \\
\hline \multicolumn{2}{|l|}{ Values are $\mathrm{n}(\%)$, mean (range), as indicated. } \\
\hline \multicolumn{2}{|c|}{ Abbreviations: DRONJ = denosumab induced medication-related osteonecrosis of the jaw. } \\
\hline
\end{tabular}

\section{Occurrence of MRONJ/DRONJ in Dmab only and Dmab+BP treated patients}

The participants were divided into two groups, one receiving only Dmab (Dmab, $\mathrm{n}=51$ [52.0\%]) and another that received BP as first treatment and Dmab as second treatment $(\mathrm{BP}+\mathrm{Dmab}, \mathrm{n}=47[48.0 \%])($ Table 2$)$. 
Table 2

Characteristics of patients treated with denosumab and bisphosphonate with extraction

\begin{tabular}{|c|c|c|c|}
\hline & Overall & $\operatorname{Dmab}^{a}(n=51)$ & $B P+D_{m a b}^{b}(n=47)$ \\
\hline \multicolumn{4}{|c|}{ Drugs administered after tooth extraction, $\mathrm{n}(\%)$} \\
\hline Denosumab & $53(54.1)$ & $37(72.5)$ & $16(34.0)$ \\
\hline Bisphosphonate & $6(6.1)$ & $2(3.9)$ & $4(8.5)$ \\
\hline Serm & $8(8.2)$ & $2(3.9)$ & $6(12.8)$ \\
\hline Teriparatide & $1(1.0)$ & . & $1(2.1)$ \\
\hline none & $30(30.6)$ & $10(19.6)$ & $20(42.6)$ \\
\hline \multicolumn{4}{|c|}{ Drug cessation to extraction, period (months) } \\
\hline $0-6, n(\%)$ & $47(64.4)$ & $15(57.7)$ & $32(68.1)$ \\
\hline $07-13, \mathrm{n}(\%)$ & $20(27.4)$ & $8(30.8)$ & $12(25.5)$ \\
\hline $14-21, \mathrm{n}(\%)$ & $6(8.2)$ & $3(11.5)$ & $3(6.4)$ \\
\hline $21-, \mathrm{n}(\%)$ & & & . \\
\hline mean $\pm S D$ & & $6.7 \pm 4.5$ & $6.9 \pm 4.8$ \\
\hline \multicolumn{4}{|c|}{ Extraction to drug initiation, period (months) } \\
\hline $0-6, n(\%)$ & $47(69.1)$ & $21(41.2)$ & $26(96.3)$ \\
\hline 07-13, n (\%) & $11(16.2)$ & $10(19.6)$ & $1(3.7)$ \\
\hline $14-21, \mathrm{n}(\%)$ & $5(7.4)$ & $5(9.8)$ & . \\
\hline $21-, n(\%)$ & $5(7.4)$ & $5(9.8)$ & . \\
\hline mean $\pm S D$ & & $7.7 \pm 9.1$ & $1.9 \pm 1.1$ \\
\hline \multicolumn{4}{|l|}{ Extraction location, n (\%) } \\
\hline Maxillary & $43(43.9)$ & $22(43.1)$ & $21(75.0)$ \\
\hline Mandible & $34(34.7)$ & $16(31.4)$ & $18(64.3)$ \\
\hline Multiple & $21(21.4)$ & $13(25.5)$ & $8(28.6)$ \\
\hline \multicolumn{4}{|c|}{ MRONJ / timing with extraction, n (\%) } \\
\hline Present & $4(4.1)$ & $1(2.0)$ & $3(6.4)$ \\
\hline Pre-extraction & $2(2.0)$ & & $2(4.3)$ \\
\hline Post-extraction & $2(2.0)$ & $1(2.0)$ & $1(2.1)$ \\
\hline Absent & $94(95.9)$ & $50(98.0)$ & $44(93.6)$ \\
\hline \multicolumn{4}{|c|}{ Values are $\mathrm{n}(\%)$ as indicated. } \\
\hline \multicolumn{4}{|c|}{ Abbreviations: MRONJ = Medication-related osteonecrosis of the jaw. } \\
\hline
\end{tabular}

In the Dmab only group, 37 (72.5\%) of the 51 patients received Dmab after tooth extraction, 2 (3.9\%) with BP, 2 (3.9\%) with Serm, and 10 patients who did not receive any drugs. The mean period from drug cessation to extraction was $6.7 \pm 4.5,15$ patients (57.7\%) within 6 months, 8 patients (30.8\%) of 7-13 months, and 3 patients (11.5\%) of 14-21 months. The mean period from tooth extraction to drug initiation was $7.7 \pm 9.1,21$ patients (41.2\%) within 6 months, 10 patients (19.6\%) of 7-13 months, 5 patients (9.8\%) of 14-21 months, and 5 patients (9.8\%) of over 21 months. The extraction site was maxillary in 22 patients (43.1\%), mandibular in $16(31.4 \%)$, and both in $13(25.5 \%)$.

In BP + Dmab, 16 (34.0\%) of the 47 patients received Dmab after tooth extraction, 4 (8.5\%) with BP, 6 (12.8\%) with Serm, 1 (2.1\%) with Teriparatide, and 20 patients (42.6\%) who did not receive any drugs. The mean period from drug cessation to extraction was $6.9 \pm 4.8,32$ patients (68.1\%) within 6 months, 12 patients $(25.5 \%)$ of $7-13$ months, and 3 patients $(6.4 \%)$ of $14-21$ months. The mean period from tooth extraction to drug initiation was $1.9 \pm 1.1,26$ patients (96.3\%) within 6 months, and 1 patients (3.7\%) of 7-13 months. The extraction site was maxillary in 21 patients (75.0\%), mandibular in 18 (64.3\%), and both in $8(28.6 \%)$.

Four of the 98 (4.1\%) patients developed MRONJ in this study. One patient belonged to the Dmab group and 3 patients belonged to the BP + Dmab group. Two of 4 patients developed MRONJ before tooth extraction, and 2 patients developed MRONJ after tooth extraction. 
Factors affecting MRONJ/DRONJ development are presented in Table 3. Among all patients, 43 (43.9\%) were in their 70s; 3 (75.0\%) patients who developed MRONJ/DRONJ were also in their 70s. 
Table 3

Occurrence of MRONJ according to drug administration and timing.

\begin{tabular}{|c|c|c|c|c|}
\hline Variables & Overall & MRONJ $(n=4)$ & NO MRONJ $(n=94)$ & $P$ value \\
\hline Age, $n(\%)$ & & & & 0.71 \\
\hline $30-39$ & $1(1.0)$ & . & $1(1.1)$ & \\
\hline $40-49$ & $1(1.0)$ & . & $1(1.1)$ & \\
\hline $50-59$ & $16(16.3)$ & $1(25.0)$ & $15(16.0)$ & \\
\hline $60-69$ & $21(21.4)$ & . & $21(22.3)$ & \\
\hline $70-79$ & 43 (43.9) & $3(75.0)$ & $40(42.6)$ & \\
\hline $80-89$ & $15(15.3)$ & . & $15(16.0)$ & \\
\hline 90- & $1(1.0)$ & . & $1(1.1)$ & \\
\hline \multicolumn{5}{|l|}{ Underlying disease, $\mathrm{n}(\%)$} \\
\hline Hypertension & $59(60.2)$ & $2(50.0)$ & $57(60.6)$ & 1.00 \\
\hline Diabetes & $31(31.6)$ & $2(50.0)$ & $29(30.9)$ & 0.26 \\
\hline Cancer & $13(13.3)$ & . & $13(13.8)$ & 0.57 \\
\hline Rheumatoid arthritis & $3(3.1)$ & $1(25.0)$ & $2(2.1)$ & 0.10 \\
\hline History of BP use, n (\%) & & & & 0.31 \\
\hline Yes & $47(48.0)$ & $3(75.0)$ & $44(46.8)$ & \\
\hline No & $51(52.0)$ & $1(25.0)$ & $50(53.2)$ & \\
\hline \multicolumn{5}{|c|}{ Timing of Dmab administration (month) } \\
\hline Pre-extraction & $73(74.5)$ & $3(75.0)$ & $70(74.5)$ & 0.97 \\
\hline within 1, n (\%) & $5(6.8)$ & $1(33.3)$ & $4(5.7)$ & \\
\hline $1, \mathrm{n}(\%)$ & $2(2.7)$ & . & $2(2.9)$ & \\
\hline $2, \mathrm{n}(\%)$ & $3(4.1)$ & . & $3(4.3)$ & \\
\hline $3, \mathrm{n}(\%)$ & $7(9.6)$ & . & $7(10.0)$ & \\
\hline $4, \mathrm{n}(\%)$ & $5(6.8)$ & . & $5(7.1)$ & \\
\hline $5, \mathrm{n}(\%)$ & $11(15.1)$ & . & $11(15.7)$ & \\
\hline $6, \mathrm{n}(\%)$ & $15(20.5)$ & $1(33.3)$ & $14(20.0)$ & \\
\hline $6-, \mathrm{n}(\%)$ & $25(34.2)$ & $1(33.3)$ & $24(34.3)$ & \\
\hline none & $20(27.4)$ & . & $20(28.6)$ & \\
\hline Post-extraction & $54(55.1)$ & $1(25.0)$ & $53(56.4)$ & 0.80 \\
\hline within 1, n (\%) & $4(4.1)$ & . & $4(4.3)$ & \\
\hline $1, \mathrm{n}(\%)$ & $10(10.2)$ & . & $10(10.6)$ & \\
\hline $2, \mathrm{n}(\%)$ & $9(9.2)$ & . & $9(9.6)$ & \\
\hline $3, \mathrm{n}(\%)$ & $5(5.1)$ & . & $5(5.3)$ & \\
\hline $4, \mathrm{n}(\%)$ & $1(1.0)$ & . & $1(1.1)$ & \\
\hline $5, \mathrm{n}(\%)$ & $3(3.1)$ & . & $3(3.2)$ & \\
\hline $6, \mathrm{n}(\%)$ & $1(1.0)$ & . & $1(1.1)$ & \\
\hline 6-, n (\%) & $20(20.4)$ & $1(100)$ & $19(20.2)$ & \\
\hline none & $1(1.0)$ & . & $1(1.1)$ & \\
\hline \multicolumn{5}{|c|}{ Values are $\mathrm{n}(\%)$, as indicated. } \\
\hline \multicolumn{5}{|c|}{ Twenty-nine of 98 participants received demosumab pre and post tooth extraction. } \\
\hline \multicolumn{5}{|c|}{ Abbreviations: $\mathrm{Bp}=$ Bisphosphonate, $\mathrm{Dmab}=$ Denosumab, MRONJ = Medication-related osteonecrosis of the jav } \\
\hline
\end{tabular}


Of 98 patients diagnosed with osteoporosis, 59 (60.2\%) had hypertension, 31 (31.6\%) had diabetes, 13 (13.3\%) had cancer, and 3 (3.1\%) had underlying rheumatoid arthritis. Among 4 patients with MRONJ, 2 had hypertension, 2 had diabetes, and 1 had rheumatoid arthritis. Three of 47 patients with a history of BP administration developed MRONJ, and 1 of 51 patients without a history of BP administration developed MRONJ. Among 98 patients, 73 (74.5\%) patients administered Dmab before tooth extraction and 54 (55.1\%) patients administered Dmab after tooth extraction. Most patients had tooth extractions $>6$ months after Dmab administration, and 1 of 3 patients who developed MRONJ had tooth extraction within 1 month after Dmab administration, 1 patient had tooth extraction 6 months later, and 1 patient had tooth extraction after $>6$ months. Among the patients who received Dmab after tooth extraction, 20 (20.4\%) patients took Dmab >6 months after tooth extraction, and one developed DRONJ >6 months after tooth extraction. There was no significant difference between the occurrence of DRONJ and factors affecting DRONJ.

\section{Case description of MRONJ/DRONJ patients}

Four patients were diagnosed with osteoporosis in this study population. Following BP and Dmab therapy, MRONJ/DRONJ developed before extraction in cases 1 and 2, and after extraction in case 3 . In case 4, the patient had no history of osteoporosis treatment at the time of extraction, and developed DRONJ afterwards, following Dmab administration 1 year after extraction (Fig. 1). Table 4 summarizes the MRONJ/DRONJ clinical cases for cases $1-4$.

Table 4

Case description of MRONJ/DRONJ of osteoporosis patient treated with

\begin{tabular}{|c|c|c|c|c|c|c|c|c|c|c|}
\hline \multicolumn{7}{|c|}{ Patients characteristics } & \multicolumn{4}{|c|}{ MRONJ/DRONJ characteristics } \\
\hline $\begin{array}{l}\text { Age } \\
\text { (years) }\end{array}$ & Sex & Underlying disease & $\begin{array}{l}\text { Previous BP } \\
\text { treatment }\end{array}$ & $\begin{array}{l}\text { Date of BP } \\
\text { treatment }\end{array}$ & $\begin{array}{l}\text { Date of } \\
\text { Dmab } \\
\text { treatment }\end{array}$ & $\begin{array}{l}\text { Date of } \\
\text { extraction }\end{array}$ & $\begin{array}{l}\text { Date of } \\
\text { DRONJ } \\
\text { diagnosis }\end{array}$ & Location & Stage & Lo \\
\hline \multicolumn{11}{|l|}{ Case 1} \\
\hline \multirow[t]{3}{*}{74} & \multirow[t]{3}{*}{ Female } & Osteoporosis & \multirow[t]{3}{*}{ Zoledronate } & $\begin{array}{l}\text { 1st: } \\
03 / 02 / 2015\end{array}$ & $\begin{array}{l}\text { 1st: } \\
10 / 16 / 2017\end{array}$ & \multirow[t]{3}{*}{ 05/28/2019 } & \multirow[t]{3}{*}{ 05/14/2019 } & \multirow[t]{3}{*}{ Mn. Rt. } & \multirow[t]{3}{*}{2} & \multirow[t]{3}{*}{$\operatorname{Prc}$} \\
\hline & & Hypercholesterolemia & & $\begin{array}{l}\text { 2nd: } \\
03 / 07 / 2016\end{array}$ & $\begin{array}{l}\text { 2nd: } \\
04 / 16 / 2018\end{array}$ & & & & & \\
\hline & & Hypertension & & & $\begin{array}{l}\text { 3rd: } \\
\text { 10/19/2018 }\end{array}$ & & & & & \\
\hline \multicolumn{11}{|l|}{ Case 2} \\
\hline 76 & Female & $\begin{array}{l}\text { Osteoporosis } \\
\text { Stable angina } \\
\text { pectoris } \\
\text { Coronary artery } \\
\text { disease }\end{array}$ & $\begin{array}{l}\text { Alendronate } \\
\text { Ibandronate } \\
\text { Zoledronate }\end{array}$ & $\begin{array}{l}\text { 1st: } \\
01 / 11 / 2007 \\
\text { 2nd: } \\
01 / 09 / 2008 \\
\text { 3rd: } \\
08 / 22 / 2017\end{array}$ & $08 / 21 / 2018$ & 03/13/2019 & $02 / 15 / 2019$ & Mx. Lt. & 1 & Pre \\
\hline \multicolumn{11}{|l|}{ Case 3} \\
\hline 54 & Female & $\begin{array}{l}\text { Osteoporosis } \\
\text { Diabetic mellitus } \\
\text { Tracheomalacia }\end{array}$ & Ibandronate & $2016^{c}$ & 04/01/2019 & 04/26/2019 & 08/22/2019 & Mn. Rt. & 2 & Ex \\
\hline \multicolumn{11}{|l|}{ Case 4} \\
\hline 79 & Female & $\begin{array}{l}\text { Osteoporosis } \\
\text { Hypertension } \\
\text { Diabetic mellitus } \\
\text { Acute myocardial } \\
\text { infarction }\end{array}$ & . & . & $\begin{array}{l}\text { 1st: } \\
\text { 07/11/2019 } \\
\text { 2nd: } \\
\text { 12/03/2019 }\end{array}$ & 07/17/2018 & $04 / 20 / 2020$ & Mn. Ant. & 2 & $\mathrm{Sp}$ \\
\hline $\begin{array}{l}\text { Abbreviation } \\
\text { a Period fron } \\
\text { b Period fron } \\
{ }^{\mathrm{c}} \text { The BP age }\end{array}$ & $\begin{array}{l}\mathrm{BP}=\text { bis } \\
\text { cessation } \\
\text { the extrac }\end{array}$ & $\begin{array}{l}\text { ohosphonate, Dmab = de } \\
\text { of Dmab therapy to extr } \\
\text { tion to the diagnosis of } \\
\text { histered from an external }\end{array}$ & $\begin{array}{l}\text { osumab, EXT } \\
\text { tion (Dmab to } \\
\text { RONJ (EXT to }\end{array}$ & $\begin{array}{l}\text { extraction, DR } \\
\text { EXT), and the } \\
\text { RONJ), and pe }\end{array}$ & $\begin{array}{l}\mathrm{NJ}=\text { denosum } \\
\text { eriod from extr } \\
\text { od from DRON }\end{array}$ & $\begin{array}{l}\text { ab induced me } \\
\text { ction to initiati } \\
J \text { diagnosis to }\end{array}$ & $\begin{array}{l}\text { ion-related ost } \\
\text { Dmab therapy } \\
\text { ction (DRONJ }\end{array}$ & $\begin{array}{l}\text { onecrosis o } \\
\text { (EXT to Dm } \\
\text { EXT). }\end{array}$ & $\begin{array}{l}\text { the jaw. } \\
\text { a). }\end{array}$ & \\
\hline
\end{tabular}

\section{Case 1}

A 74-year-old woman had rheumatoid arthritis, hypercholesterolemia, and hypertension and a history of zoledronic acid (5 mg/100mg) therapy from March 2015 to March 2016 due to osteoporosis. From October 2017 to October 2018, she received a total of three Dmab doses. In December 2018 , there was severe tooth mobility due to localized chronic advanced periodontitis, and extraction was planned to be performed after $\geq 3$ to 6 months. Conservative treatment was 
performed before extraction, but MRONJ was diagnosed in the right mandibular region 6 months after the last Dmab administration. The drug was changed to Calcitriol [0.25 mcg/ Soft Cap], and extraction and sequestrectomy were performed after lesion localization (Supplementary Fig. S1 online).

\section{Case 2}

A 76-year-old woman had stable angina pectoris and coronary artery disease, apart from osteoporosis, was treated with endodontics and prosthesis for a crown and root fracture that had occurred approximately 10 years prior. From January 2007 to May 2017, she was administered alendronate and ibandronate, and in August 2017, she was administered zoledronic acid (5 mg/100mg). One year later, Dmab was administered once. Six months thereafter, MRONJ occurred in the left maxillary region in the presence of localized chronic advanced periodontitis, and bone necrosis was observed up to the mesial root site from the first premolar to the second molar in the radiographic image. Approximately 4 weeks later, tooth extraction and sequestrectomy of the affected area were performed, followed by prosthetic rehabilitation of the missing teeth (Supplementary Fig. S2 online).

\section{Case 3}

A 54-year-old woman had diabetes mellitus, osteoporosis, and a history of ibandronate (Bonviva [3 mg/ syringe]) treatment at a different hospital in 2016. In April 2019, Dmab was administered once. Within a month, the mandibular right second molar had a hopeless prognosis due to a fracture in the tooth, and the tooth was extracted. Three months after extraction, necrotic bone exposure (MRONJ) was found on the lingual side of the extraction site. With Dmab clearing up around October 2019, the area was observed during follow-up and treated conservatively (Supplementary Fig. S3 online).

\section{Case 4}

A 79-year-old woman with underlying diseases such as hypertension, diabetes mellitus, and acute myocardial infarction, apart from osteoporosis, did not undergo any osteoporosis treatment before tooth extraction. With the removal of partial dentures used $>10$ years, the mandibular anterior teeth had severe mobility and periodontic-endodontic lesions. The teeth were extracted in July 2018. Dmab was administered twice 11 months after the extraction, and bone loss and increased sclerosis were observed in the anterior mandible in April 2020, 10 months after Dmab administration. Conservative treatment was performed by changing Dmab by raloxifene (60 mg/T) in June 2020 (Supplementary Fig. S4 online).

\section{Discussion}

Various factors have been implicated as causes of MRONJ, but recently, it was suggested that anti-resorptive agents affect the immune function of the bone. Tooth extraction has been reported as a predisposing factor of MRONJ in approximately $45-61 \%$ cases, but the prevalence and incidence rates of other spontaneous occurrences, which are the second largest factor and present as periodontal or periapical lesions, implants, or dentures, as well as extractions, have not been reported ${ }^{12,13}$. In animal studies, lesions similar to ONJ have been reported in cases of periapical inflammation and periodontitis ${ }^{7,14}$. However, there is still a lack of clinical research on Dmab-induced MRONJ and its incidence, and no protocol has been established yet for invasive dental treatment of the patients receiving this drug.

In this study, we reported four cases of MRONJ/DRONJ, out of 98 patients who received either only Dmab injections or with BP and who underwent tooth extractions. Although all four cases have something in common, i.e., they are cases of MRONJ/DRONJ occurring after Dmab administration, each situation is distinct. Three of the four DRONJ cases (Cases 1-3) had a history of BP treatment. As for predisposing events, Cases 1- 3 can be considered tooth extraction due to periodontal disease, and case 4 as a natural occurrence due to an existing pathogen. Over time, case 1 and 2 resulted in MRONJ due to worsening of the chronic inflammation of the existing lesion after the Dmab administration, regardless of tooth extraction. This means that chronic active periodontitis may have been a significant risk factor in the two cases, suggesting the importance of preventive dental treatment. For the MRONJ related to an existing lesion, the prevalence rate of BP-induced MRONJ is $28.6 \%$, but that for Dmab-induced MRONJ has not yet been reported ${ }^{15}$.

Several hypotheses have been raised regarding the transformation of existing chronic inflammatory lesions into MRONJ, and an M1 macrophage shift is considered the most likely explanation. Kang et al. in 2013, Aghaloo et al. in 2014, and Soundia et al. in 2016, reported the development of osteonecrosis when zoledronate or a RANKL inhibitor was administered to mice after inducing pulp disease by drilling molar teeth ${ }^{16-18}$. Cheong et al. observed an increase in BP uptake at the apex in mice with periapical disease, and suggested that this may be associated with ONJ development ${ }^{19}$. In addition, in 2011 , Aghaloo et al. reported the occurrence of osteonecrosis when administering zoledronate in a rat periodontitis model with ligature ${ }^{20}$. In other words, osteonecrosis occurs when anti-resorptive drugs, such as zoledronate, are administered in the presence of pulpal and periodontal disease, which are representative oral inflammatory diseases. Recently, Kim et al. reported that the incidence of osteonecrosis decreases if pre-inflammatory conditions are removed prior to extraction and emphasized the importance of pulpal and periodontal disease as local factors in the occurrence of MRONJ ${ }^{14}$.

Osteonecrosis occurs when BP or RANKL inhibitors are administered in the presence of inflamed pulpal or periodontal tissue, because anti-resorptive agents affect the function of various immune cells, such as of neutrophils and polymorphonuclear leukocytes, macrophages, and dendritic cells ${ }^{21-27}$

Kyrgidis et al. reported a change in the distribution of monocytes in peripheral blood samples of patients treated with Dmab, which could alter the immune system ${ }^{28}$. Numerous studies have also investigated their relationship with dendritic cells. Elsayed et al. reported that zoledronate impairs dendritic cell function, which eliminates bacteria and adversely affects $T$ cell effector response ${ }^{[21}$. Kalyan et al. reported that $y \delta T$ cells decreased significantly in patients who received $\mathrm{BP}$ and that this may be closely related to the occurrence of $\mathrm{ONJ}^{22}$.

The most recently studied immune cells are macrophages, and a particular focus on macrophage polarization. Polarization refers to the different ratio of M1 macrophages having pro-inflammatory and antimicrobial properties and M2 macrophages having anti-inflammatory properties ${ }^{29,30}$. Zhang et al. reported that 
IL-17 mediated M1/M2 macrophage alteration is related to the development of BRONJ ${ }^{23}$, Tamaki et al. reported that dynamic M1/M2 macrophage polarization was induced by the anti-RANKL antibody in a MRONJ model ${ }^{31}$, while Weber et al. analyzed MRONJ in Wistar rats and found that zoledronate induces a macrophage M1 shift ${ }^{24}$.

Human studies on macrophage function in this context have also been performed. For instance, Hoefert et al. reported that patients with MRONJ had a compromised macrophage function when compared to patients with osteoradionecrosis and osteomyelitis ${ }^{25}$, and Paschalidi et al. found M1/M2 macrophage polarization in macrophages of patient tissues in MRONJ, and that macrophages shift to the M1 phenotype at stages 2 and 3 , as compared to stage 1 of MRONJ ${ }^{26}$.

In Case 3, the extraction was performed one month after the administration of a single Dmab dose following ibandronate injection. DRONJ developed three months after surgery. The lesion was classified as grade 2 DRONJ, and cured with a five-month conventional treatment. This case suggests a one month time between the Dmab injection and extraction may be too short.

The patient in Case 4 had no history of BP administration, and the development of DRONJ was "non-identified" after two administrations of Dmab a year after extraction. This non-identified occurrence is peculiar in that the possibility of a pathological fracture in the jawbone during Dmab treatment cannot be ruled out. The most common causes of MRONJ are tooth extraction and obvious periodontal disease, followed by "non-identified" occurrences with no identifiable cause $^{15}$. This led to the consideration of systemic or local causes that could have other effects other than on the onset. Nevertheless, an MRONJ case associated with a predisposing event (i.e., "non-identified") is the third most common group of MRONJ cases reported in the literature, with an estimated prevalence between $16-70 \%{ }^{32}$. Most of these non-identified occurrences were found to occur in relation to the lower posterior tooth region, but the lesion described in Case 4 developed anteriorly. Importantly, a non-identified MRONJ occurrence poses the risk of a pathological fracture of the jaw after Dmab administration.

This study provides information on the incidence of MRONJ/DRONJ in patients with Dmab but has limitations. First, it was a retrospective study using data from a single institution. In addition, bone turnover markers (BTM) were not investigated because all participants' BTMs were not measured at the same time. However, clinical data only makes it difficult to predict the occurrence of DRONJ and requires another predictive factor, therefore molecular biomarkers need to be included in future research.

In conclusion, the incidence of DRONJ after Dmab administration was $4.1 \%$, and the timing of drug administration and release varied in our study population. DRONJ can occur as a chronic inflammation without invasive dental treatment, therefore it is necessary to reduce its likelihood by implementing preventive dental treatment before Dmab treatment.

\section{Declarations}

\section{ACKNOWLEDGEMENTS}

This work was supported by the National Research Foundation of Korea (NRF) grant funded by the Korean government (MSIT) (No. 2020R1I1A1A0107285612).

\section{AUTHOR'S CONTRIBUTIONS}

Study design and conduct: WP and HJK. Data collection: JYK, Data analysis: JYK, SJ and WP. Data interpretation: JYK, SJ, JHP, HJK and WP. Writing initial draft: JYK and SJ. Writing-review and editing: JHP, HJK and WP. Approving final version of manuscript: All authors.

\section{COMPETING INTERESTS}

JaeYeon Kim, Seoyeon Jung, Jin Hoo Park, Hyung-Jun Kim and Wonse Park declare that they have no competing interests.

\section{DATA AVAILABILITY}

The datasets generated during and/or analysed during the current study are available from the corresponding author on reasonable request.

\section{References}

1. Seeman, E. \& Martin, T. J. Antiresorptive and anabolic agents in the prevention and reversal of bone fragility. Nat. Rev. Rheumatol. 15, 225-236 (2019).

2. Yusuf, A. A. et al. Real-world effectiveness of osteoporosis therapies for fracture reduction in post-menopausal women. Arch. Osteoporos. 13, 33 (2018).

3. Everts-Graber, J., Reichenbach, S., Ziswiler, H. R., Studer, U. \& Lehmann, T. A single infusion of zoledronate in postmenopausal women following denosumab discontinuation results in partial conservation of bone mass gains. J. Bone Miner. Res. 35, 1207-1215 (2020).

4. Doria, C. et al. Denosumab is really effective in the treatment of osteoporosis secondary to hypogonadism in prostate carcinoma patients? A prospective randomized multicenter international study. Clin. Cases Miner. Bone Metab. 13, 195-199 (2016).

5. Nicolatou-Galitis, O. et al. Medication-related osteonecrosis of the jaw: definition and best practice for prevention, diagnosis, and treatment. Oral Surg. Oral Med. Oral Pathol. Oral Radiol. 127, 117-135 (2019).

6. Govaerts, D. et al. Adjuvant therapies for MRONJ: A systematic review. Bone 141, 115676 (2020). 
7. Ruggiero, S. L. et al. American Association of Oral and Maxillofacial Surgeons position paper on medication-related osteonecrosis of the jaw - 2014 update. J. Oral Maxillofac. Surg. 72, 1938-1956 (2014).

8. Tanna, N., Steel, C., Stagnell, S. \& Bailey, E. Awareness of medication related osteonecrosis of the jaws (MRONJ) amongst general dental practitioners. Br. Dent. J. 222, 121-125 (2017).

9. Marx, R. E. Pamidronate (Aredia) and zoledronate (Zometa) induced avascular necrosis of the jaws: a growing epidemic. J. Oral Maxillofac. Surg. 61, 1115-1117 (2003).

10. Kajizono, M. et al. Incidence and risk factors of osteonecrosis of the jaw in advanced cancer patients after treatment with zoledronic acid or denosumab: A retrospective cohort study. Biol. Pharm. Bull. 38, 1850-1855 (2015).

11. Lee, C. Y. \& Suzuki, J. B. CTX biochemical marker of bone metabolism. Is it a reliable predictor of bisphosphonate-associated osteonecrosis of the jaws after surgery? Part II: A prospective clinical study. Implant Dent. 19, 29-38 (2010).

12. Saad, F. et al. Incidence, risk factors, and outcomes of osteonecrosis of the jaw: integrated analysis from three blinded active-controlled phase III trials in cancer patients with bone metastases. Ann. Oncol. 23, 1341-1347 (2012).

13. McGowan, K., McGowan, T. \& Ivanovski, S. Risk factors for medication-related osteonecrosis of the jaws: A systematic review. Oral Dis. 24, 527-536 (2018).

14. Kim, T. et al. Removal of pre-existing periodontal inflammatory condition before tooth extraction ameliorates medication-related osteonecrosis of the jawlike lesion in mice. Am. J. Pathol. 188, 2318-2327 (2018).

15. Marx, R. E., Sawatari, Y., Fortin, M. \& Broumand, V. Bisphosphonate-induced exposed bone (osteonecrosis/osteopetrosis) of the jaws: risk factors, recognition, prevention, and treatment. J. Oral Maxillofac. Surg. 63, 1567-1575 (2005).

16. Kang, B. et al. Periapical disease and bisphosphonates induce osteonecrosis of the jaws in mice. J. Bone Miner. Res. 28, 1631-1640 (2013).

17. Aghaloo, T. L. et al. RANKL inhibitors induce osteonecrosis of the jaw in mice with periapical disease. J. Bone Miner. Res. 29, 843-854 (2014).

18. Soundia, A. et al. Osteonecrosis of the jaws (ONJ) in mice after extraction of teeth with periradicular disease. Bone 90, 133-141 (2016).

19. Cheong, S. et al. Bisphosphonate uptake in areas of tooth extraction or periapical disease. J. Oral Maxillofac. Surg. 72, 2461-2468 (2014).

20. Aghaloo, T. L. et al. Periodontal disease and bisphosphonates induce osteonecrosis of the jaws in the rat. J. Bone Miner. Res. 26, 1871-1882 (2011).

21. Elsayed, R. et al. Role of dendritic cell-mediated immune response in oral homeostasis: A new mechanism of osteonecrosis of the jaw. FASEB J. 34, 2595-2608 (2020).

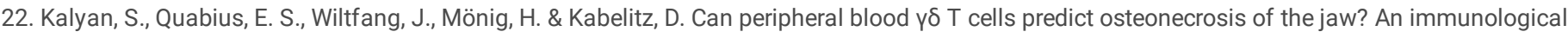
perspective on the adverse drug effects of aminobisphosphonate therapy. J. Bone Miner. Res. 28, 728-735 (2013).

23. Zhang, Q. et al. IL-17-mediated M1/M2 macrophage alteration contributes to pathogenesis of bisphosphonate-related osteonecrosis of the jaws. Clin. Cancer Res. 19, 3176-3188 (2013).

24. Weber, M. et al. Zoledronate causes a systemic shift of macrophage polarization towards M1 in vivo. Int. J. Mol. Sci. 22 (2021).

25. Hoefert, S., Schmitz, I., Weichert, F., Gaspar, M. \& Eufinger, H. Macrophages and bisphosphonate-related osteonecrosis of the jaw (BRONJ): evidence of local immunosuppression of macrophages in contrast to other infectious jaw diseases. Clin. Oral Investig. 19, 497-508 (2015).

26. Paschalidi, P. et al. The role of M1 and M2 macrophage polarization in progression of medication-related osteonecrosis of the jaw. Clin. Oral Investig. 25, 2845-2857 (2021).

27. Hayano, H. et al. Distinct immunopathology in the early stages between different antiresorptives-related osteonecrosis of the jaw-like lesions in mice. Bone 135, 115308 (2020).

28. Kyrgidis, A. et al. Changes in peripheral monocyte populations $48-72$ hours after subcutaneous denosumab administration in women with osteoporosis. J. Musculoskelet. Neuronal Interact. 20, 339-346 (2020).

29. Shapouri-Moghaddam, A. et al. Macrophage plasticity, polarization, and function in health and disease. J. Cell. Physiol. 233, 6425-6440 (2018).

30. Yunna, C., Mengru, H., Lei, W. \& Weidong, C. Macrophage M1/M2 polarization. Eur. J. Pharmacol. 877, 173090 (2020).

31. Tamaki, S. et al. Dynamic polarization shifting from M1 to M2 macrophages in reduced osteonecrosis of the jaw-like lesions by cessation of anti-RANKL antibody in mice. Bone 141, 115560 (2020).

32. Khominsky, A. \& Lim, M. "Spontaneous" medication-related osteonecrosis of the jaw; two case reports and a systematic review. Aust. Dent. J. 63 , $441-454$ (2018).

\section{Figures}




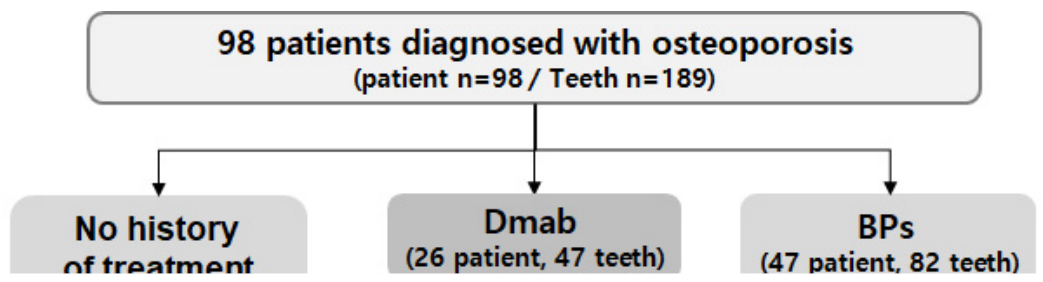

Figure 1

Flow of DRONJ occurrence. Drug administration and DRONJ route before and after tooth extraction in 98 patients diagnosed with osteoporosis. A total of four patients developed DRONJ. In two of these patients, DRONJ occurred after BP and Dmab administration and before extraction and after extraction in another patient. The remaining patient had undergone extraction without prior osteoporosis treatment, and then DRONJ developed after administration of Dmab.

Abbreviations: $\mathrm{BP}=$ bisphosphonate, Dmab = denosumab, EXT = extraction, DRONJ = denosumab induced medication-related osteonecrosis of the jaw. The dotted line represents the DRONJ flow. ${ }^{a}$ Teriparatide agents and selective estrogen receptor modulators (raloxifene or bazedoxifene).

\section{Supplementary Files}

This is a list of supplementary files associated with this preprint. Click to download.

- supplementaryfigure.pdf 